\title{
EDITORIAL
}

\section{G-proteins and the lung: nuts and bolts and out of the gutter}

\author{
C. Kroegel*, W. Luttmann, J.C. Virchow, H. Matthys
}

The first documented description of inflammation as a distinct phenomenon dates back several centuries B.C. to preserved Mesopotamian clay tablets and Egyptian medical papyri [1]. Since then, through the eminent work by J.F. Cohnheim, E. Mechnikoff, T. Leber and many others thereafter, as well as the advances in modern molecular biology, the understanding of mechanisms underlying inflammation has made much progress. At the present time, inflammation may be adequately described as an extremely complex series of interdigitating processes, in a wide variety of combinations and permutations, which is presumably designed as a reparative and protective response to external noxae and tissue injury.

The cornerstones of this dynamic pathophysiological host reaction are a variety both of circulating and constitutive cells, which communicate through an even more complex web of structural or soluble signals. To complicate matters further, it has become apparent that the net biological effect of intercellular messengers, or mediators, may be determined by microenvironmental factors and varies depending on the composition, as well as the state of degradation, of the surrounding matrix, the actual concentration of a particular signal achieved at tissue level, and the presence or absence of co-secrated mediators [2]. In addition, the biological outcome may ultimately depend on the state of activation and/or maturation of the individual target cell, which, in turn, may be regulated both by the cell itself (autocrine action), and other surrounding or distant cells (paracrine action). However, the principal molecular mechanisms by which the cell discriminates between the plethora of synergistic or antagonistic incoming signals have long remained obscure.

When, during the 1950s, Sutherland discovered that epinephrine exerts its effects by stimulating the intracellular generation of a then unknown compound called cyclic adenosine monophosphate (cAMP), he unveiled the first of many secondary messengers [3]. It was for this discovery, that Sutherland received the 1971 Nobel prize. Although the precise mechanism by which epinephrine binding to its respective receptor might stimulate cAMP was unknown, it was generally assumed that the receptor directly signalled the cAMP-generating enzyme adenylate cyclase. However, as more cAMP-

*Correspondence: C. Kroegel, Dept of Pneumology, Medical Clinic, Albert-Ludwigs University, Hugstetter Str. 55, D-79106 Freiburg, Germany. stimulating receptors and secondary messengers were discovered, it became clear that this receptor-secondary messenger model could not account for the diversity in exogenous signals on the one hand and the cellular response on the other. It was left to the work of biochemist M. Rodbell of the National Institute of Environmental Health Sciences in Research Triangle Park, North Carolina, and the pharmacologist A.G. Gilman of the University of Texas Southwestern Medical Center in Dallas to discover another missing piece in the puzzle, which bridges the multitude of surface receptors and intracellular signalling pathways $[4,5]$ ultimately determining the individual cellular response in a given context.

The cellular mechanisms of the physiological regulation have been the subject of intense research for some time, and certain principles of cellular pathways have emerged throughout the past decade or so. Utilization of intracellular calcium, cAMP and diacylglycerol (DAG), as well as the protein kinase cascades and the inositol phosphate cycle, represent well-established examples [6]. In addition, the interactive crosstalk of the major cell signalling systems at different levels between plasma membrane and gene transcription and cell cycle control are beginning to be unveiled. Due to the groundwork by Gilman and Rodbell, there is now substantial evidence that an increasing number of ubiquitous proteins, which are located close to the inner aspect of the cell membrane, may serve as a highly versatile molecular switch that regulate the seemingly infinite external signals and cellular signalling processes. According to their characteristic property of binding guanine nucleotides, they have collectively been termed Gproteins.

G-proteins belong to a larger superfamily of guanosine triphosphases (GTPases) that include both factors controlling protein synthesis, of which the elongation factor-Tu (EF-Tu) has been studied most extensively, and a group of "small" guanosine triphosphate (GTP)binding proteins with a molecular mass of 20-25 kD. Members of the latter group include the ras gene product, the adenosine diphosphate (ADP)-ribosylation factor (ARF) (a co-factor for cholera toxin catalysed ADP-ribosylation of $\mathrm{G}_{\mathrm{s}}$ ), $\mathrm{G}_{\mathrm{p}}$ (a GTP-binding protein purified from the placenta) the rho gene product, and products of yeast YPT1 and SEC4 genes.

In contrast to the small G-proteins, classical G-proteins are heterotrimers composed of three distinct subunits 
termed $\alpha, \beta$ and $\gamma$, in order of decreasing mass [7]. The subunits $\beta$ and $\gamma$ are tightly associated and function as a unit. In the basal state, this subunit complex favours the association with a guanosine diphosphate (GDP)carrying $\alpha$-subunit. During activation of G-proteins, GTP is substituted for GDP at the binding site on the $\alpha$-subunit, a site that is structurally conserved across the entire superfamily, which results in the dissociation of the heterotrimer leaving GTP- $\alpha$ and free B $\gamma$-subunits. Whilst the $\beta \gamma$-subunit forms a new heterotrimer with another GDP- $\alpha$ unit, the original $\alpha$-subunit slowly converts GTP to GDP via the action of its intrinsic hydrolytic activity, eventually returning the G-protein to its inactive heterotrimeric form.

The family of G-protein $\alpha$-units can be divided according to their functional or genetic structural relationships. As yet, 21 distinct $\alpha-$, four $\beta$-, and six $\gamma$-subunits have been cloned, and this number is sure to rise [7]. According to their structural homologies, $\alpha$-subunits can be subclassified into four subfamilies $G_{s}, G_{i}, G_{q}$, and $G_{12}$. Functionally, each member of these subfamilies link receptors to different intracellular signalling cascades in different cell types, but members of different subfamilies may also propagate the signal to the same secondary messenger system. For instance, whereas hormone or odorant receptors interact with members of the $\mathrm{G}_{\mathrm{s}}$ family to stimulate adenylate cyclase, members of the $G_{i}$ family are involved both in inhibition of adenylate cyclase and activation or inhibition of various ion channels following exposure to several hormones. In addition, members of the $\mathrm{G}_{\mathrm{q}}$ family transduce the actions of a broad spectrum of hormones, neurotransmitters and growth hormones to phospholipase $\mathrm{C}$ in various organs.

G-Protein-linked receptors have been identified for several classes of drugs and mediators believed to play a role in respiratory physiology, pathophysiology and pharmacology, such as neurotransmitters, neuropeptides, and the phospholipid-derived mediators. For instance, there is ample evidence that G-proteins may play a role in regulating the neurovegetative regulation of the airway muscle tone, and other functions such as mucus secretion and mucociliary clearance. G-proteins have been identified and cloned for the $\beta_{1}, \beta_{2}, \beta_{3}$ as well as $\alpha_{1}$ and $\alpha_{2}$ adrenoreceptors, the five subtypes of muscarinic receptors $M_{1}$ to $M_{5}$, and for the $A_{1}$ and $A_{2}$ adenosine-receptors. Beta-agonist receptors are coupled to a $G_{s}$ protein containing the $\alpha_{s}$-subunit, which propagates the signal across the membrane to stimulate adenylate cyclase, thereby increasing intracellular cAMP levels. In contrast, activation of the $\alpha_{2}$-adrenoreceptor inhibits the adenylate cyclase via a $\mathrm{G}_{1}$ protein employing the $\alpha_{i 1}$-subunit.

Muscarinic cholinergic (MCho) receptor stimulation, which produces bronchial secretion and the contraction of bronchial smooth muscle, is processed through a number of different G-proteins. For instance, $M_{1}$ Cho receptors are present in the parasympathetic ganglia, and may regulate not only secretion of gastric acid but also the vagal tone in asthmatic airways [8]. $\mathrm{M}_{1}$ Cho receptors, like $\alpha_{1}$-adrenoreceptors, are linked to $\mathrm{G}_{\mathrm{q}}$ containing an $\alpha_{\mathrm{q}}$-subunit, which propagates activation of phospholipase $\mathrm{C}$, resulting in phosphatidylositol turnover and the release of calcium from intracellular calcium stores. In contrast, the $\mathrm{M}_{2}$ Cho receptor is located at the terminals of cholinergic nerves and is connected to a $\mathrm{G}_{\mathrm{i}}$ protein containing $\alpha_{\mathrm{i} 2}$ as the GTP-binding unit. Stimulation of $\mathrm{M}_{2}$ Cho receptors inhibit adenylate cyclase and modulate ion channels for both $\mathrm{K}^{+}$and $\mathrm{Ca}^{++}$. $\mathrm{M}_{2} \mathrm{Cho}$ receptors serve as autoreceptors and attenuate acetylcholine release from neurons via a negative feedback loop. They are believed to limit cholinergic bronchoconstriction following inhalation of sulphur dioxide $\left(\mathrm{SO}_{2}\right)$. In asthma, there is some evidence that $\mathrm{M}_{2} \mathrm{Cho}$ receptors may be dysfunctional [8], a finding which is believed to contribute to the pathogenesis in $\beta$-blockerinduced asthma [9]. Thus, the molecular defect in this condition may reside within the G-protein-dependent signal transduction.

Whilst major scientific interest focuses around the $\alpha$ subunits of G-proteins as the nucleotide binding units with intrinsic GTPase enzyme activity, there is some circumstantial evidence that the $\beta \gamma$-subunit complex also participates in functions other than stabilization of the GDP- $\alpha$-subunit, receptor presentation and membrane anchor for the obligomer. For instance, in mammalian brain cells, several types of adenylate cyclase are inhibited by $\beta \gamma$-dimers, albeit solely in the presence of $\mathrm{G}_{\mathrm{s} \alpha}$ [7]. This constellation may come about when the dimer would arise by dissociation of $G_{i}$ following $\alpha_{2}-$ adrenergic stimulation, whilst $\mathrm{G}_{\mathrm{sa}}$ would be liberated by activation of $\mathrm{G}_{\mathrm{s}}$-linked $\beta$-adrenergic receptors. More importantly, such a situation highlights the possibility of crosstalk between different signalling pathways via G-proteins. In addition, the fact that $\beta \gamma$-subunits can bind to several members of $\alpha$-unit subclasses possibly provide another differential communication pathway between second messenger systems.

A new family of GTP-binding proteins has recently been identified. The prototype for this family is the ras oncoprotein, p21ras, but well over 30 related proteins are now known, and that figure is rising. All are monomeric and around $21-25 \mathrm{kD}$ in size, bind GDP/ GTP, and have an instrinsic GTPase activity. The ras protein is localized at the inner side of the plasma membrane, and besides transducing extracellular signals into cellular responses it is essentially involved in regulating normal cell growth [10]. In addition, there is some evidence that proteins of the ras gene may contribute to the malignant transformation underlying the development of human lung tumours. There are two principal ways through which ras proto-oncogenes may acquire transforming potential: 1) overexpression of an otherwise normal gene; or 2) through a point mutation in specific codons that change ras proteins [10].

Three mutant protein products of the ras oncogene, designated $\mathrm{K}$-ras, H-ras and $\mathrm{N}$-ras, have been identified. Instead of cycling back and forth from the active ras: GTP to the inactive ras:GDP form these mutations "lock" the protein into its active GTP-bound conformation. As a result, the cell receives a constitutive and uncontrolled proliferative signal, one of the hallmarks of a malignant cell. Some 300 lung cancers have now 
been studied by three different groups [11-13], and more than $30 \%$ of adenocarcinoma as well as $20 \%$ of the large-cell carcinoma demonstrated a ras gene mutation. In contrast, ras mutants have only been identified occasionally in squamous cell or adenosquamous carcinoma, and never in small cell carcinoma. In addition, studies with resected stage I and II adenocarcinomas as well as with more advanced cancers show that patients whose tumours exhibit a ras mutation have a significantly poorer prognosis $[11,12]$. The reasons for the raised mortality are not clear, but ras mutations correlate highly with cigarette smoking [14].

Although at present, information about ras mutations is of prognostic use only, understanding of the ras regulatory pathway may furnish new types of adjuvant therapies. For instance, both the GTPase-activating protein p120 GAP and the product of the neurofibromatosis-1, NF1 gene-regulated protein (NF-1GRP) have been shown to stimulate the intrinsic GTPase activity of p21 ras [15, 16]. These proteins appear to be responsible for facilitating the conversion of GTP-bound ras to the inactive GDP-bound ras. Thus, p120 GAP and NF1GRP, respectively, may exert anti-oncogene effects via downregulation of p21ras mutant proteins.

Although less is known about other proteins of the ras superfamily, there is some evidence that they are also involved in regulating granulocyte effector functions. For instance, in macrophages and neutrophils, p21 rac regulates the assembly of nicotinonide-adenine dinucleotide phosphate (reduced form) (NADPH) oxidase at the plasma membrane [17], suggesting a regulatory role of G-proteins in the respiratory burst of inflammatory cells. In addition, members of the $r a b$ sub-family of small G-proteins have been shown to facilitate endo- and exocytosis of cells. $r a b$ proteins are localized to particular intracellular organelles, and their active GTP-bound forms are believed to be involved in targeting cytoplasmic vesicles by recognizing a docking protein complex on the plasma membrane [18]. Further-more, a recent study suggests that a number of small GTP-binding proteins are associated with lung lamellar bodies, the organelles responsible for the secretion of surfactant molecules by type II pneumocytes [19]. Although these investigations were performed in the rate, the findings indicate that Gproteins may contribute both to uptake and secretion of surfactants in the lung.

The bestowal of this years Nobel prize for physiology and medicine to A.G. Gilman and M. Rodbell throws the G-proteins out of the gutter and into the limelight, not only for basic researchers, but also for the pneumologist and pulmonary pharmacologist. Considering the increasing number of G-protein subunits identified, and the potential permutations of the trimeric G-proteins as well as the high number of still poorly understood guanine nucleotide-dependent cellular responses, it appears to be most likely that more G-protein-regulated pathways will be discovered in the future. Possible candidates include phospholipase $\mathrm{A}_{2}$, phospholipase $\mathrm{D}$, several ion channels, transporters and exchangers. In addition, there is evidence that a single G-protein $\alpha$-subunit may transduce more than one effector [7], suggesting that the assignment of a given G-protein to a specific receptor-effector pathway may be too simplistic. This also implies that the other regulatory mechanisms may modify the G-protein pathway itself. Moreover, unveiling the role of G-proteins may improve understanding of how drugs interact. Furthermore, they may form the basis for different response rates of drug treatment in patients, and could help to answer the question as to why one drug is more beneficial in a given disorder than in another. Finally, a more detailed understanding of the regulation or alteration of G-proteins may provide new insights into the principles of oncogenesis. In years to come deciphering the nuts and bolts of G-proteins will significantly increase our insights into how the array of different mediators will shape an individual cellular response in various physiological and pathophysiological conditions encompassing lung disease.

\section{References}

1. Manjo G. Inflammation and infection: historic highlights in current topics. In: Manjo G, Cotran C, Kaufman L, eds. Inflammation and Infection, Baltimore, Williams and Wilkins, 1982; pp. 1-17.

2. Elias JA, Zitnik RJ. Cytokine-cytokine interactions in context of cytokine networking. Am J Respir Cell Mol Biol 1992; 7: 365-367.

3. Robinson GA, Butcher RW, Sutherland EW. Cyclic AMP. New York, Academic Press, 1977.

4. Rodbell M, Birnbaum L, Pohl SL, Krans HMJ. The glucagon-sensitive adenyl cyclase system in plasma membrane of rat liver. V. An obligatory role of guanyl nucleotides in glucagon action. J Biol Chem 1971; 246: 1877-1882.

5. Ross EM, Gilman AG. Resolution of some components of adenylate cyclase necessary for catalytic activity. $J$ Biol Chem 1977; 252: 6966-6969.

6. Nishizuka Y. Signal transduction: crosstalk. TIBS 1992; 17: 367.

7. Hepler JR, Gilman AG. G-proteins. TIBS 1992; 17: 383-387.

8. Barnes PJ. Muscarinic receptors in the airway: recent developments. J Appl Physiol 1990; 68: 1777-1785.

9. Barnes PJ. Neural mechanisms in asthma. Br Med Bull 1992; 48: 149-168.

10. Hall A. The cellular functions of small GTP-binding proteins. Science 1990; 249: 635-640.

11. Slebos R. Kibbelaar O, et al. K-ras oncogene activation as a prognostic marker in adenocarcinoma of the lung. $N$ Engl J Med 1990; 323: 561-565.

12. Mitsudomi T, Steinberg S, Oie H, et al. Gene mutations in non-small cell cancers are associated with shortened survival irrespective of treatment intent. Cancer Res 1991; 51: 4999-5002.

13. Rodenhuis S, van de Wetering ML, Mooi WJ, et al. Mutational activation of the H-ras oncogene: a possible pathogenetic factor in adenocarcinoma of the lung. $N$ Engl J Med 1987; 317: 929-935.

14. Rodenhuis S, Slebos R, Boot AJM, et al. Incidence and possible clinical significance of K-ras oncogene activation in adenocarcinoma of the human lung. Cancer Res 1988; 48: 5738-5741.

15. Ballester R, Marchuck D, Boguski M, Saulino A, Letcher 
R. The NF1 locus encodes a protein functionally related to mammalian GAP and yeast IRA proteins. Cell 1990; 63: 851-859.

16. Xu GF, Lin B, Tanaka K, et al. The catalytic domain of neurofibromatosis type 1 gene product stimulates ras GTPase and complement ira mutants of S. cerevisiae. Cell 1990; 63: 835-841.

17. Abo A, Pick E, Hall A, Totty N, Teahan CG, Segal AW Activation of the NADPH oxidase involves the small
GTP-binding protein P21rac. Nature 1991; 353: 668670.

18. Balch WE. Small GTP-binding proteins in vesicular transport. Nature 1990; 353: 668-670.

19. Rubins JB, Panchenko M, Shannon TM, Dickey BF. Identification of ras- and ras-related low molecular mass GTP-binding proteins associated with rat lung lamellar bodies. Am J Respir Cell Mol Biol 1992; 6: 253259. 